\title{
717 SELECTIVE ACTIVATION OF CD8+ T CELLS BY A CD8- TARGETED IL-2 RESULTS IN ENHANCED ANTI-TUMOR EFFICACY AND SAFETY
}

${ }^{1}$ Kelly Moynihan, 'Danielle Pappas, ${ }^{1}$ Terrence Park, 'Wei Chen, 'Irene Ni, ${ }^{1}$ Paul Bessette, ${ }^{1}$ Mike Chin, ${ }^{2}$ Ton Schumacher, ${ }^{1}$ Andy Yeung, ${ }^{1}$ Ivana Djuretic*. ${ }^{1}$ Asher Biotherapeutics, South San Francisco, CA, United States; ${ }^{2}$ The Netherlands Cancer Institute, Amsterdam, Netherlands

Background High-dose IL-2 induces complete responses in a subset of cancer patients, but severe toxicity, including vascular leak syndrome (VLS), limits its clinical potential. Insights into IL2R $\alpha$ 's role in the development of VLS sparked a wave of second-generation IL-2 molecules referred to as "not- $\alpha$ " IL-2s. Emerging clinical data suggests that although not- $\alpha$ IL-2s avoid VLS, they induce suboptimal monotherapy activity in patients. Given the observation that $\mathrm{CD} 8+\mathrm{T}$ cells are the dominant effector cells with IL-2-based therapies, ${ }^{1}{ }^{2}$ we hypothesized that maximizing the activity of IL-2 on CD8 + T-cells and limiting its activity on immunosuppressive Tregs and highly IL-2sensitive innate populations would improve IL-2's efficacy and tolerability. We developed cis-targeted IL-2 (CD8-IL2) fusion proteins that selectively activate CD $8+\mathrm{T}$ cells and have minimal activity on CD8-negative cells.

Methods In vitro selectivity of CD8-IL2 molecules was tested on primary immune cells including mouse splenocytes and human PBMCs. In vivo activity was evaluated in syngeneic tumor models and non-human primates.

Results Due to the $10-20 \mathrm{x}$ higher expression of IL2R $\beta$ on NK cells over other lymphocytes, not- $\alpha$ IL-2 induced preferential NK cell expansion in mice. Toxicity-induced body weight loss with not- $\alpha$ IL-2 treatment was dependent on cells expressing NK1.1 but not CD8. To avoid overt activation of IL2Rßhigh NK cells, IL-2R $\alpha$-associated toxicity, and Treg activation, we generated cis-targeted fusion proteins consisting of anti-CD8 antibodies and IL-2 muteins with attenuated binding to IL2R $\alpha$ and IL2R $\beta$. We demonstrated that CD8-IL2 fusions preferentially activated CD8 $+\mathrm{T}$ cells within mouse, human, and cynomolgus immune populations, with 100-1000 fold selectivity over NK cells and Tregs for all three species. Selective expansion of CD8 $+\mathrm{T}$ cells over NK cells and Tregs was demonstrated in tumor and peripheral blood compartments in mice. Selective CD8 $+\mathrm{T}$ cell expansion was also demonstrated in cynomolgus monkeys. Furthermore, a single dose of CD8IL2 in mice elicited strong monotherapy efficacy in MC38 tumors, with a majority of mice demonstrating complete responses without detectable body weight loss at doses that were well tolerated in cynomolgus monkeys. In contrast, not alpha IL-2 induced $>10 \%$ body weight loss prior to reaching efficacious doses in mice and did not drive any complete antitumor responses.

Conclusions CD8-targeted IL-2 has superior efficacy and lower toxicity compared to second-generation not- $\alpha$ IL-2. Development of AB248, a novel CD8-targeted IL-2 molecule is underway.

\section{REFERENCES}

1. Rakhmilevich A, North R. Elimination of CD4+ T cells in mice bearing an advanced sarcoma augments the antitumor action of interleukin-2. Cancer Immunol Immunother 1994:38(2):107-12.

2. Sun Z, Ren Z. A next-generation tumor-targeting IL-2 preferentially promotes tumor-infiltrating CD8+ T-cell response and effective tumor control. Nat Commun 2019;3874:1-12

http://dx.doi.org/10.1136/jitc-2021-SITC2021.717 